\title{
AN INVESTIGATION INTO ALIASING IN IMAGES RECAPTURED FROM AN LCD MONITOR USING A DIGITAL CAMERA
}

\author{
Hani Muammar and Pier Luigi Dragotti \\ Electrical and Electronic Engineering Department, Imperial College London, United Kingdom
}

\begin{abstract}
With current technology, high quality recaptured images can be created from soft displays, such as an LCD monitor, using a digital still camera and professional image editing software. The task of verifying the ownership and past history of an image is, consequently, more difficult. One approach to detecting an image that has been recaptured from an LCD monitor is to search for the presence of aliasing due to the sampling of the monitor pixel grid. To validate this approach, an investigation into the aliasing introduced in a digitally recaptured image is conducted. An anti-forensic method for recapturing images that are free from aliasing is developed using a model of the image acquisition process. This is supported by a simulation of the acquisition process and illustrated with examples of recaptured images that are free from aliasing.
\end{abstract}

Index Terms - forensic imaging, recapture, Bayer CFA, digital camera

\section{INTRODUCTION}

Over the last decade advances in digital camera technology have meant that high resolution images with excellent colour fidelity can easily be obtained at relatively low cost to the consumer. With the widespread availablity of high quality softcopy display mediums, such as LCD monitors and projection displays, an image can be reproduced with ease by recapturing it from a display using a digital camera. Cao and Kot [1] studied the ability of observers to identify recaptured images from LCD monitors. They reported that good quality recaptured images are often difficult to distinguish from originally captured images. The integrity and authenticity of an image, therefore, comes into question. Image recapture can, for example, be used to disguise artefacts introduced in images that have been tampered with or manipulated using image editing software.

The problem of detecting and identifying recaptured images from LCD monitors has received interest from researchers in recent years [1] [2]. Aliasing due to the sampling

This work is supported by the REWIND Project, funded by the future and Emerging Technologies (FET) programme within the 7th Framework Programme for Research of the European Commission, FET Open grant number: 268478

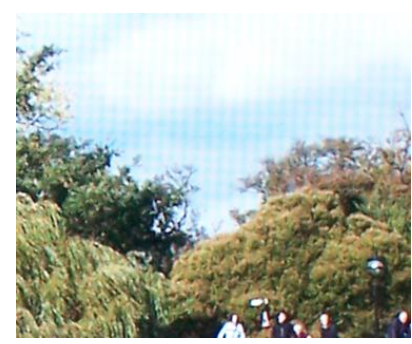

(a) Recaptured image

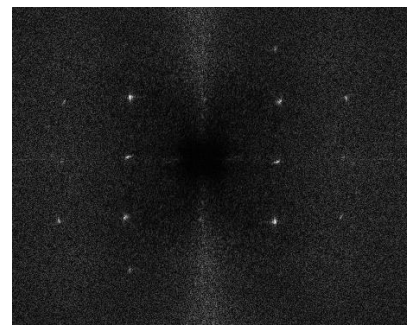

(b) DFT of noise residual
Fig. 1. Example of recaptured image showing (a) aliasing and (b) the DFT of its noise residual

of the monitor pixel structure is a feature commonly seen in recaptured images. It is unlikely to exist in originally captured images and is very difficult to eliminate by postprocessing [3]. Most digital cameras, today, are fitted with a Bayer colour filter array (CFA) to enable colour images to be obtained using a single image sensor. To generate a full colour image the data from the filtered sensor are interpolated using a demosaicking algorithm [4]. The type of the demosaicking algorithm used can affect the level of aliasing that is introduced into the interpolated image. Although research in the area of demosaicking algorithms has led to novel approaches that reduce the level of false colour introduced in aliased regions of the interpolated image [5] [6] [7], aliasing remains a problem when recapturing the high frequency patterns that exist in an LCD monitor. One way of detecting the presence of aliasing in recaptured images is to search for peaks in the 2D DFT of the noise residual that are associated with the periodic structures present in the image due to the aliasing. The noise residual can be formed by taking the difference between the recaptured image and a denoised version of the image. An example of an image recaptured from an LCD monitor and the DFT of its noise residual is shown in Figure 1.

Before a robust solution to recapture detection based on aliasing can be developed, we need to establish whether it is possible to eliminate aliasing under certain capture conditions. A method for reducing the level of moiré in images recaptured from an LCD monitor using a digital camera was described by Hatada and Saitoh [8]. Their method, however, 
required that two images at different focus positions are captured. Cao and Kot [1] claimed that by adjusting camera exposure, capture mode, zoom and focus distance, elimination of the 'visible texture pattern' was possible. They stated, however, that complete elimination of the pattern was very difficult. In this paper we show that aliasing can be completely eliminated in a recaptured image by setting the camera to monitor distance to a value determined by the camera lens focal length, the pixel pitch of the LCD monitor and the pixel pitch of the camera's image sensor. A recapture detector should not therefore rely solely on the presence of aliasing, but should make use of other features present in recaptured images such as high scene tonal contrast, changes in colour balance and loss in perceived sharpness.

In Section 2 an overview of aliasing in cameras that use the Bayer CFA is provided and a description of how the monitor pixel grid can be modelled in one dimension by a sampled $2 \mathrm{D}$ square wave is given. We show how aliasing can be eliminated in the recaptured image in Section 3 and in Section 4 the model is validated by examples of images recaptured from an LCD screen and through a simulation of the acquisition process.

\section{ALIASING IN RECAPTURED IMAGES}

\subsection{Digital cameras and the Bayer CFA}

Aliasing can be introduced in a digitally acquired image when frequency components from the replicated components during sampling enter the Nyquist boundary of the sampled system. This can occur when the scene that is being captured is insufficiently band-limited.

In a digital camera aliasing is commonly referred to as chroma moiré. A schematic diagram of the Bayer CFA pattern arrangement, used in most digital cameras today, is shown in Figure 2(a). The arrangement of pixels in the green channel of a Bayer array can be described by a quincunx lattice. As can be seen from Figure 2(b) the horizontal and vertical Nyquist frequency response of the green channel is the same as the response of the native unfiltered sensor, however, in the diagonal direction the location of the Nyquist frequency is at $(0.25,0.25)$ [9]. The red and blue channels are sampled on a rectangular lattice at twice the sampling period of the native sensor. Because of the horizontal and vertical offsets of the red and blue sampling lattices, respectively, relative to the green lattice, the Fourier transforms of the red and blue channels are out of phase at the replication points, $(0,0.5)$ and $(0.5,0)$, shown in Figure 2(b). Thus, aliasing in the red and blue channel results in the orange-cyan pattern commonly seen in aliased images captured with a Bayer CFA [10]. Aliasing in all three channels will result in green-magenta patterns. To limit aliasing digital camera manufacturers fit optical anti-aliasing filters [3] but these are often weak in high end cameras thereby increasing the risk of

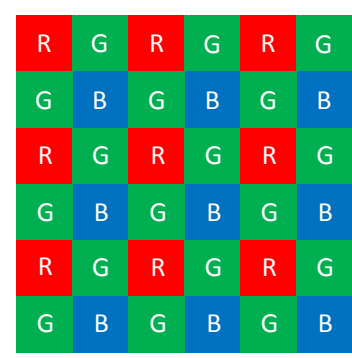

(a) Bayer CFA schematic

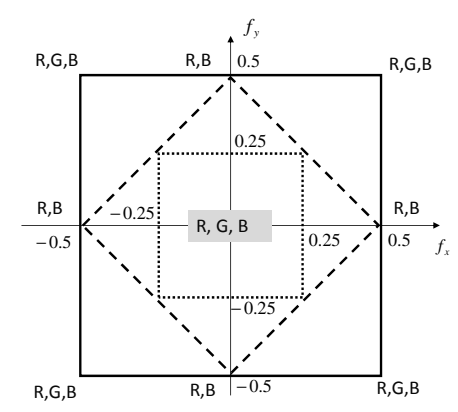

(b) Nyquist boundary
Fig. 2. The Bayer CFA pattern (a) and a graph (b) showing the Nyquist boundary for the unfiltered sensor (solid), green (dashed) and red/blue channels (dotted) of the Bayer CFA. Replication points for the red (R), green $(\mathrm{G})$ and blue (B) channels are shown. All frequencies are in cycles/pixel.

visible aliasing artefacts in the captured image.

\subsection{Modelling of the Monitor Pixel Grid}

In a modern twisted nematic (TN) LCD monitor each pixel comprises three long and narrow vertically oriented sub-pixel elements that are covered with red, green and blue filters. The monitor pixel pitch, $T_{m}$, is defined as the distance between adjacent pixel elements. In the horizontal direction this corresponds to the distance between two successive equal coloured sub-pixel elements. Normally the pixel pitch of the monitor is the same in the horizontal and vertical dimensions.

When capturing a monitor with a digital camera the pixel pitch of the monitor as projected on the image sensor, $T_{o}$, is given by: $T_{o}=m T_{m}$, where $m$ is the optical magnification of the camera lens. Note that throughout the analysis we have assumed that camera lens geometric distortions are negligible and that the monitor is fronto-parallel to the image sensor plane. The luminance signal generated from the projection of the LCD monitor pixel grid is periodic with period equal to $T_{o}$. We can model the monitor pixel grid in the vertical direction by an infinite $2 \mathrm{D}$ square wave pattern ${ }^{1}$ as shown in Figure 3(a). The frequency spectrum of the square wave can be found by taking the Fourier transform of its Fourier series and can be expressed as [11]:

$$
G\left(f_{x}, f_{y}\right)=\sum_{n_{o}=-\infty}^{\infty} C_{n_{o}} \delta\left(f_{x}-\frac{n_{o}}{T_{o}} \cos \theta_{o}, f_{y}-\frac{n_{o}}{T_{o}} \sin \theta_{o}\right)
$$

where, for a square wave, $C_{n_{o}}=\sin \left(\pi n_{o} / 2\right) /\left(\pi n_{o}\right)$. The frequency spectrum of the sampled image is given by the convolution of the spectrum of the square wave with the camera sampling system. The delta functions are distributed at the

\footnotetext{
${ }^{1}$ The model may be extended to the horizontal dimension of the monitor pixel grid by rotating the 2D square wave pattern by $90^{\circ}$.
} 


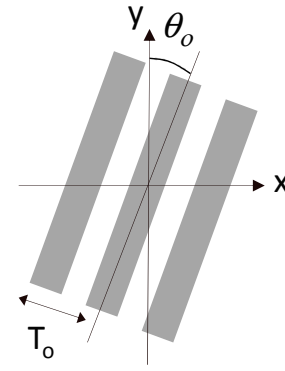

(a) 2D Square Wave

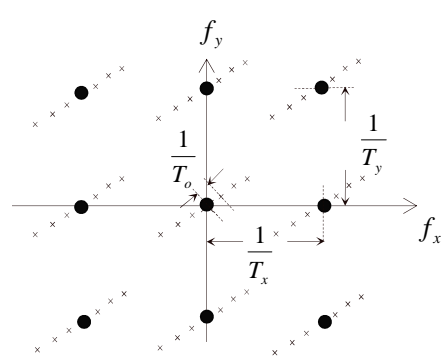

(b) Fourier transform
Fig. 3. 2-D square wave model of monitor pixel grid and the Fourier transform of the sampled square wave

locations given by the vectors:

$$
\left(f_{x}, f_{y}\right)=\left(\frac{n_{o}}{T_{o}} \cos \theta_{o}+\frac{n_{x}}{T_{x}}, \frac{n_{o}}{T_{o}} \sin \theta_{o}+\frac{n_{y}}{T_{y}}\right),
$$

for $n_{o}, n_{x}$ and $n_{y}=-\infty \ldots-1,0,1 \ldots \infty$. The parameters $T_{x}$ and $T_{y}$ correspond to the horizontal and vertical image sensor pixel pitch dimensions respectively. Thus the spectrum of the square wave exists at DC and is replicated at $n_{x} / T_{x}$ and $n_{y} / T_{y}$. A schematic representation of the spectrum is shown in Figure 3(b). Aliasing will occur when delta functions associated with the replicated spectrum with high enough amplitude fall within the Nyquist boundary limit defined by the $\left[1 / 2 T_{x}, 1 / 2 T_{y}\right]$.

\section{CANCELLATION OF ALIASING}

Aliasing is not visible in the recaptured image when delta functions from the replicated spectra are either located inside the Nyquist boundary but their amplitudes are weak, or they lie at the exact locations of the delta functions associated with the spectrum centred at DC. The former condition holds when the camera sampling period is much smaller than the monitor pixel pitch, and low pass filtering is applied, perhaps by slightly defocusing the lens at capture. The latter condition exists when the monitor pixel pitch projected on the sensor is an integer multiple of the sensor pixel pitch. Thus, on the horizontal axis of the pixel grid, $k=T_{o} / T_{x}$, where $k$ is a positive integer value. Considering the case where the monitor pixel grid is perfectly aligned to the sensor pixel grid (i.e. $\theta_{o}$ is zero), and substituting $k T_{x}$ for $T_{o}$ in equation (2), the location of the delta functions is then described by:

$$
\left(f_{x}, f_{y}\right)_{g}=\left(\frac{n_{o}}{k T_{x}}+\frac{n_{x}}{T_{x}}, \frac{n_{y}}{T_{y}}\right) .
$$

Equation (3) is true for an unfiltered sensor and for the green channel of the Bayer CFA. Aliasing, therefore, is eliminated in the green channel when $T_{o}=k T_{x}$. Since the red and blue channels are sampled on a rectangular lattice at twice the sampling period, the location of the delta functions of the sampled monitor pixels that have been filtered by the red or blue components of the Bayer CFA is given by:

$$
\left(f_{x}, f_{y}\right)_{r, b}=\left(\frac{n_{o}}{k T_{x}}+\frac{n_{x}}{2 T_{x}}, \frac{n_{y}}{2 T_{y}}\right) .
$$

Thus, aliasing is eliminated in all channels in the recaptured image when the period of the monitor pixel pitch, projected on the image sensor is an even multiple of the sensor pixel pitch. That is, $T_{o}=2 k T_{x}$, where $k$ is a positive integer.

To determine the projected size of the monitor pixel on the image sensor during capture, we need to know the camera lens magnification, $m$. From Gaussian optics [12], we can express the distance between the camera sensor and the LCD monitor, $d$, as a function of lens focal length, $f$, and magnification as:

$$
d=-\frac{f(m-1)^{2}}{m} .
$$

Substituting $m T_{m}$ for $T_{o}$ we can express the condition for alias cancellation during recapture as $m T_{m}=2 k T_{x}$. Rearranging in terms of $m$ and since by convention, magnification is negative since the projected image size on the sensor is smaller than the object size, we get $m=-2 k T_{x} / T_{m}$. Substituting the expression for $m$ into equation (5) and simplifying, we obtain:

$$
d=2 f\left(\frac{k T_{x}}{T_{m}}+\frac{T_{m}}{4 k T_{x}}+1\right) .
$$

Hence, for any integer value, $k$, equation (6) may be used to determine the required camera to monitor distance to obtain a recapture that is free of aliasing. If we make the assumption that the camera and monitor pixels are square then aliasing is eliminated in both the horizontal and vertical axes of the recaptured image. Note that the ratio of projected monitor pixel pitch to image sensor pixel pitch is equal to $2 k$. For a good quality recapture, a value of $k=1$, or two projected monitor pixels to a sensor pixel, is recommended. The camera is likely to resolve the monitor pixels at values of $k$ greater than 1 resulting in a low quality recapture.

\section{MODEL VALIDATION}

Images of a natural scene were displayed on a HP LA1905wg 19" LCD monitor and recaptured using a Kodak V550 compact 5 megapixel digital camera. To maximise the degree of control over the recapture settings a Kaiser RS10 copy stand was used. The monitor was fitted to an adjustable platform and positioned on its back on the base of the copy stand. Prior to recapture the monitor was adjusted so that its front panel was fronto-parallel to the camera's image sensor and the monitor pixel grid aligned with the image sensor pixel grid. All acquisitions were conducted in a darkened room to eliminate any specular reflections from the front panel of the monitor. A series of test exposures of a dot grid pattern was used to 


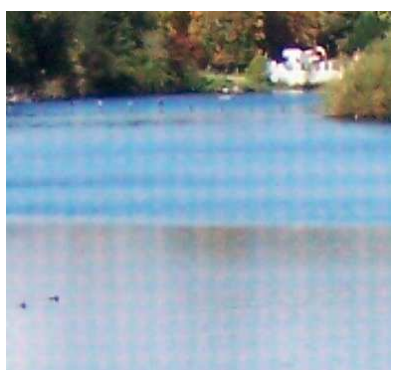

(a) Recaptured image with aliasing

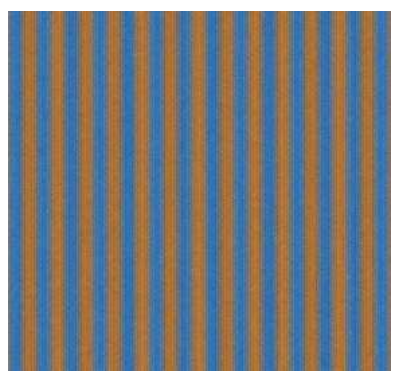

(b) Simulated acquisition
Fig. 4. Example of recaptured image with aliasing

determine a range of preset camera focal length settings that introduced acceptably low levels of geometric distortion into the captured image. The actual focal length of the lens was then determined for each setting. The use of a copy stand enabled the camera to monitor distance to be varied without significantly altering the other parameter settings. To determine the pixel pitch of the sensor used in the Kodak V550, the sensor width and height were divided by the number of pixels in the horizontal and vertical directions, respectively. The average value was determined as $2.228 \times 10^{-3} \mathrm{~mm}$. The pixel pitch for the monitor reported by the manufacturer was $0.2835 \mathrm{~mm}$.

A simulation of the image acquisition path for the recapture of an LCD monitor with a digital camera was also implemented. The simulation gave us a higher degree of flexibility compared to performing actual screen recaptures and confirmed that the aliasing obtained in real images is due to the phenomenon observed. A synthetic $2 \mathrm{D}$ target square wave pattern with vertical and horizontal dimensions 16 times greater than the dimensions of the image sensor was generated. The high resolution synthetic target was blurred to simulate the effect of the lens point spread function and optical anti-aliasing filter by convolving it with a Gaussian filter. The image data were then averaged over adjacent square neighbourhoods and sampled at intervals corresponding to the locations of the pixels in the image sensor. Gaussian distributed zero mean noise was added to simulate both temporal and fixed pattern sensor noise sources. The Bayer CFA pattern was applied to generate sparsely sampled, red, green and blue components of the sampled target. A full colour image was generated by demosaicking the CFA data using a bi-linear interpolation algorithm.

The monitor screen was recaptured from a distance of $840 \mathrm{~mm}$ with the camera lens focal length set to $14.1 \mathrm{~mm}$. In Figure 4(a) we show an example of a poorly recaptured image where aliasing is clearly visible in the cropped region of the recaptured image. The same aliasing pattern is visible in Figure 4(b) where the same reacquisition process has been simulated, thus confirming the validity of our analysis. We note that aliasing with a frequency of 0.0475 cycles/pixel in

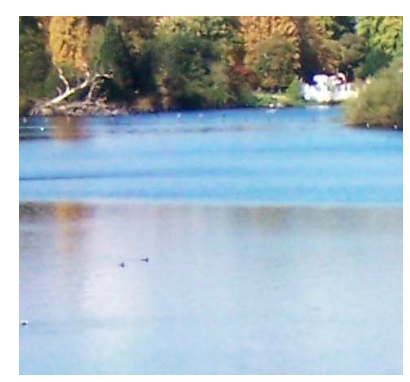

(a) Alias free recaptured image

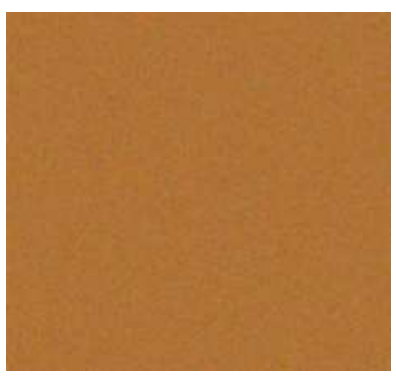

(b) Simulated acquisition
Fig. 5. Example showing a recapture that is free from aliasing

the red and blue channel is present. This is confirmed by the orange-cyan banding that is present in both the original recapture and the simulation where aliasing with a periodicity of approximately 21 pixels was estimated. The delta functions from the replicated spectra that lie inside the Nyquist boundary in the green channel are too weak to cause visible aliasing in the recaptured image.

To recapture an image free from aliasing artefacts, the camera focal length was set to $11.58 \mathrm{~mm}$. Using equation (6) and a value of $k=1$ a camera to monitor distance of $760 \mathrm{~mm}$ was determined. The resulting alias free recaptured image is shown in Figure 5(a). The simulation result, shown in Figure 5(b) contains no aliasing and is orange in colour due to the blue and red signals being out of phase and the mean code value of the blue signal being far lower than red. The DFT of the recaptured image noise residual was evaluated and was found to contain no discernible peaks.

Additional examples of recaptures with and without aliasing can be found at the url given by: http://www.commsp.ee. ic.ac.uk/ hmuammar/ICASSP13/recaptured_images.zip

\section{CONCLUSION}

An analysis of aliasing in recaptured images of LCD monitors using digital cameras equipped with a Bayer CFA was presented. The periodic structure of the monitor pixel grid projected on the camera's image sensor was modelled in one dimension by a 2-dimensional square wave. It was shown that aliasing in the recaptured image can be cancelled by setting the camera to monitor distance to a value determined by the camera's focal length and the monitor and image sensor pixel pitch dimensions. Examples of images with and without aliasing were presented and a simulation of the recapture acquisition process supported the results obtained. A database of recaptured images that will include alias free images is currently being developed and will be made publicly available when complete. It is anticipated that the database will form a valuable resource for the development and benchmarking of forensic methods for the detection of recaptured images from LCD monitors. 


\section{REFERENCES}

[1] Hong Cao and A.C. Kot, "Identification of recaptured photographs on LCD screens," in IEEE International Conference on Acoustics Speech and Signal Processing (ICASSP), 2010, March 2010, pp. 1790-1793.

[2] Jing Yin and Yanmei Fang, "Digital image forensics for photographic copying," in Proceedings of the SPIE - The International Society for Optical Engineering, 2012, vol. 8303, p. 83030F (7 pp.).

[3] Rastislav Lukac, Ed., Single-Sensor Imaging: Methods and Applications for Digital Cameras (Image Processing Series), chapter 4, pp. 105-135, CRC Press, 1st edition, September 2008.

[4] B.K. Gunturk, J. Glotzbach, Y. Altunbasak, R.W. Schafer, and R.M. Mersereau, "Demosaicking: color filter array interpolation," Signal Processing Magazine, IEEE, vol. 22, no. 1, pp. 44 - 54, Jan. 2005.

[5] D. Alleysson, S. Susstrunk, and J. Herault, "Linear demosaicing inspired by the human visual system," Image Processing, IEEE Transactions on, vol. 14, no. 4, pp. 439 -449, April 2005.

[6] Xin Li, B. Gunturk, and Lei Zhang, "Image demosaicing: a systematic survey," in Proceedings of the SPIE - The International Society for Optical Engineering, 2008, vol. 6822, pp. $68221-1$.

[7] M. Aghagolzadeh, A.A. Moghadam, M. Kumar, and H. Radha, "Compressive demosaicing for periodic color filter arrays," in 2011 18th IEEE International Conference on Image Processing (ICIP 2011), 2011, pp. 16936.

[8] T. Hatada and F. Saitoh, "Moire reduction method for LCD captured image by using two different focused images," IEEJ Transactions on Electronics, Information and Systems, vol. 128, pp. 326-327, 2008.

[9] Nai-Xiang Lian, Lanlan Chang, Yap-Peng Tan, and V. Zagorodnov, "Adaptive filtering for color filter array demosaicking," Image Processing, IEEE Transactions on, vol. 16, no. 10, pp. 2515 -2525, Oct 2007.

[10] K. Topfer, J.E. Adams, and B.W. Keelan, "Modulation transfer functions and aliasing patterns of cfa interpolation algorithms," in Society for Imaging Science and Technology: Image Processing, Image Quality, Image Capture, Systems Conference, 1998, pp. 367 - 370.

[11] J. Krumm and S.A. Shafer, "Sampled-grating and crossed-grating models of moire patterns from digital imaging," Optical Engineering, vol. 30, no. 2, pp. 195206, 1991.
[12] Warren J Smith, Modern Optical Engineering: the design of optical systems, chapter 2, pp. 21-33, McGraw Hill: New York, 4th edition, 2008. 